\title{
Improved theory for the polarization-dependent transverse shift of a paraxial light beam in free space
}

\author{
Bekshaev A.
}

I.I. Mechnikov National University, 2 Dvorianska St., 65082 Odessa, Ukraine, e-mail: bekshaev@onu.edu.ua

Received: 10.11 .2010

\begin{abstract}
Spatial distribution of longitudinal field component of circularly polarised optical beam depends on the polarization handedness, which causes a lateral shift of "centre of gravity" of the beam when its polarization toggles. We present generalised theory of this effect, which demonstrates relation of the latter with angular irradiance moments of the beam. The theory is applicable to arbitrary paraxial beams and shows that the lateral shift is the same for the all cross sections of the beam.
\end{abstract}

Keywords: paraxial beam, circular polarization, transverse shift

PACS: 42.25.-p, 42.25.Ja, 42.90.+m

UDC: $535.131,535.47,535.51$

\section{Introduction}

Processes in which polarization state affects spatial characteristics of a light beam are known as manifestations of spin-orbital interaction of light [1-13]. Such effects can take place in the presence of inhomogeneous or anisotropic media [2-8] as well as in free space [9-13]. One of the most spectacular manifestations of the spin-orbit coupling is a "spin Hall effect of light", a polarization-induced transverse shift of the beam trajectory. Usually it is expressed by displacement of "centre of gravity" (CG) of the transverse energy distribution in the beam, depending on the handedness of its circular polarization $[2-6,9-11,13]$. In the classical version, this phenomenon is local and associated with strong inhomogeneity occurring, e.g., at a plane boundary between different optical media [3-6]. However, a similar effect may take place with freely propagating optical fields (in particular, upon tight focusing of a perturbed Gaussian beam [11]). Then it cannot be attributed to a certain beam section and relates to the whole length of the beam [14], i.e. is non-local.

Historically, the first effect of this sort has been discovered in 1994 when the polarization-dependent shift of the focal spot along $y$-direction has been predicted [9] and observed [10] in the conditions of focusing an initially collimated (paraxial) beam whose transverse profile is asymmetric in the $x$ direction (Fig. 1). This shift has been explained 
by employing longitudinal $(z)$ component of the electric vector of the beam electromagnetic field whose spatial distribution shows explicit relation to the polarization handedness. However, the explanation given in [9] seems insufficient for the two reasons:

(i) it relies essentially on a special model of the incident beam which is supposed to be of the shape of Hermite-Gaussian mode [15];

(ii) formulation of Ref. [9] produces misleading impression as if the transverse shift were formed "on the passage" of light from the lens to the focal plane, and, when observing the focal plane image it cannot be seen because "at the stage of divergent wave propagation from the focal plane towards the microscope objective lens the effect of the opposite sign will identically nullify that shift" [9]. This assertion evidently contradicts the known fact that the beam CG propagates along the geometric-optics trajectory, which is a straight line in the free space after the focusing lens.

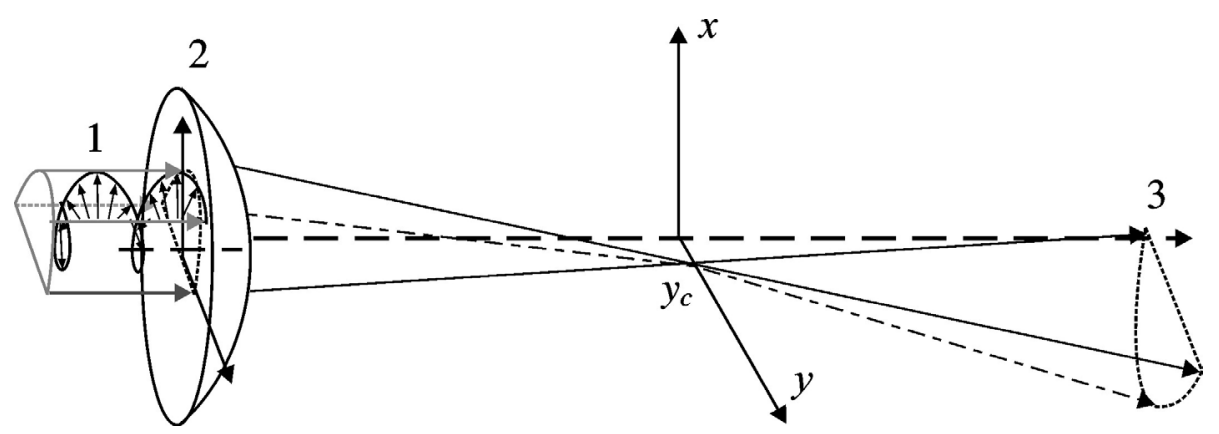

Fig. 1. Scheme of experiment for detecting polarization-dependent shift of a focal spot [10]: a circularly polarised beam 1 is focused by lens 2 , Cartesian frame $(x, y)$ parameterises the focal plane, and inverse image of cross section of the incident beam is formed in the image plane 3 . An asymmetry along $x$ direction is created by screening a lower half of lens 2 . The focal spot shift $y_{c}$ along $y$ direction is shown.

In fact, exhaustive description of the beam behaviour in this situation must rely on non-paraxial models recently developed basing on the plane-wave expansion of the light field [14, 16]. However, following Ref. [9], a consistent quantitative picture of the effect can be achieved by relatively simple means employing only paraxial concepts. In this brief report we present a generalization of the approach [9], which is applicable to arbitrary incident beams. Simultaneously, we intend to show that the same effect takes place in arbitrary cross section behind the lens, and that exclusive role of the focal plane is caused solely by a small transverse size of the focal spot. The absolute value of the transverse shift of the beam CG is the same everywhere but the tiny effect can be seen easier for a "narrow" focal spot than for any other cross sections of the focused beam.

\section{Basic analysis}

Let us consider a monochromatic paraxial beam propagating along a $z$ axis. Its electromagnetic field can be represented as a superposition of $X$ - and $Y$-polarised components with the electric and magnetic vectors $[15,17]$ 


$$
\begin{gathered}
\left\{\begin{array}{c}
\mathbf{E}_{X} \\
\mathbf{H}_{X}
\end{array}\right\}=\exp (i k z)\left(\left\{\begin{array}{c}
\mathbf{e}_{x} \\
\mathbf{e}_{y}
\end{array}\right\} u_{X}+\frac{i}{k} \mathbf{e}_{z}\left\{\begin{array}{c}
\partial / \partial x \\
\partial / \partial y
\end{array}\right\} u_{X}\right), \\
\left\{\begin{array}{c}
\mathbf{E}_{Y} \\
\mathbf{H}_{Y}
\end{array}\right\}=\exp (i k z)\left(\left\{\begin{array}{c}
\mathbf{e}_{y} \\
-\mathbf{e}_{x}
\end{array}\right\} u_{Y}+\frac{i}{k} \mathbf{e}_{z}\left\{\begin{array}{c}
\partial / \partial y \\
-\partial / \partial x
\end{array}\right\} u_{Y}\right),
\end{gathered}
$$

where $u_{j}(j=X, Y)$ are slowly varying complex amplitudes. The longitudinal components of the field given by Eq. (1) are

$$
E_{z}=\frac{i}{k}\left(\frac{\partial u_{X}}{\partial x}+\frac{\partial u_{Y}}{\partial y}\right), \quad H_{z}=\frac{i}{k}\left(-\frac{\partial u_{Y}}{\partial x}+\frac{\partial u_{X}}{\partial y}\right) .
$$

In frame of the paraxial approximation, the longitudinal field given by Eqs. (2) is small with respect to the transverse field, $E_{z} \sim \gamma\left(E_{x}, E_{y}\right)$, where the small parameter $\gamma$ coincides with the angle of self-diffraction (beam divergence) [17].

The energy density of the beam electromagnetic field can be presented as

$$
w=\frac{1}{16 \pi}\left(|\mathbf{E}|^{2}+|\mathbf{H}|^{2}\right)=w_{\perp}+w_{z},
$$

where the first summand,

$$
w_{\perp}=\frac{1}{8 \pi}\left(\left|u_{X}\right|^{2}+\left|u_{Y}\right|^{2}\right),
$$

originates from the transverse components of the field (1), while the contribution associated with the longitudinal field is

$$
w_{z}=\frac{1}{16 \pi}\left(\left|E_{z}\right|^{2}+\left|H_{z}\right|^{2}\right) \sim \gamma^{2} w_{\perp} .
$$

Our aim is to inspect modification of the beam transverse position caused by changes of the incident beam polarization. This position is characterised by the CG of the transverse energy distribution:

$$
\mathbf{r}_{c}=\frac{\int \mathbf{r} w d x d y}{\int w d x d y}
$$

where $\mathbf{r}=\left(\begin{array}{l}x \\ y\end{array}\right)$ is the transverse radius-vector. From now on, all integrations are performed over the whole cross section of the beam. In agreement with Eq. (3), we can separate the contributions of $\mathbf{r}_{c}$ due to the longitudinal and transverse field components: $\mathbf{r}_{c}=\mathbf{r}_{c \perp}+\mathbf{r}_{c z}$, with

$$
\mathbf{r}_{c \perp}=\frac{\int \mathbf{r} w_{\perp} d x d y}{\int w d x d y} \approx \frac{\int \mathbf{r} w_{\perp} d x d y}{\int w_{\perp} d x d y}
$$

and 


$$
\mathbf{r}_{c z}=\frac{\int \mathbf{r} w_{z} d x d y}{\int w d x d y} \approx \frac{\int \mathbf{r} w_{z} d x d y}{\int w_{\perp} d x d y} .
$$

The second equalities in Eqs. (7) and (8) are possible due to small relative value of $w_{z}$ (see Eqs. (3) and (5)). Now notice that in the beams with homogeneous polarization, for which

$$
u_{Y}=\beta u_{X}
$$

with an arbitrary complex constant $\beta$, the constituent $\mathbf{r}_{c \perp}$ in Eq. (7) does not depend on the polarization. This means that all the polarization influences are accumulated in the expression given by Eq. (8). Considering Eqs. (2) and (5) and accounting for smallness of $w_{z} / w$, we can represent the term under interest as a sum of two summands,

$$
\mathbf{r}_{c z}=\mathbf{r}_{c 1}+\mathbf{r}_{c 2},
$$

where

$$
\begin{gathered}
\mathbf{r}_{c 1}=\frac{1}{2 k^{2} I} \int \mathbf{r}\left(\left|\frac{\partial u_{X}}{\partial x}\right|^{2}+\left|\frac{\partial u_{X}}{\partial y}\right|^{2}+\left|\frac{\partial u_{Y}}{\partial x}\right|^{2}+\left|\frac{\partial u_{Y}}{\partial y}\right|^{2}\right) d x d y, \\
\mathbf{r}_{c 2}=\frac{1}{2 k^{2} I} \int \mathbf{r}\left(\frac{\partial u_{X}^{*}}{\partial x} \frac{\partial u_{Y}}{\partial y}-\frac{\partial u_{X}^{*}}{\partial y} \frac{\partial u_{Y}}{\partial x}+\frac{\partial u_{Y}^{*}}{\partial y} \frac{\partial u_{X}}{\partial x}-\frac{\partial u_{Y}^{*}}{\partial x} \frac{\partial u_{X}}{\partial y}\right) d x d y,
\end{gathered}
$$

and

$$
I=\int\left(\left|u_{X}\right|^{2}+\left|u_{Y}\right|^{2}\right) d x d y .
$$

Again, the component (11) does not depend on $\beta$ under the condition (9) and is thus invariant with respect to the polarization. Therefore, it is the term (12) that expresses the sought spin Hall effect in the considered situation, and so it will be in the focus of our further attention. Integration by parts, with taking into account that $u_{X}(x, y), u_{Y}(x, y)$ and all their derivatives vanish at the transverse infinity, directly gives

$$
\begin{gathered}
\int x \frac{\partial u_{X}^{*}}{\partial x} \frac{\partial u_{Y}}{\partial y} d x d y=-\int\left(u_{X}^{*} \frac{\partial u_{Y}}{\partial y}+x u_{X}^{*} \frac{\partial^{2} u_{Y}}{\partial x \partial y}\right) d x d y, \\
-\int x \frac{\partial u_{X}^{*}}{\partial y} \frac{\partial u_{X}}{\partial y} d x d y=\int x u_{X}^{*} \frac{\partial^{2} u_{Y}}{\partial x \partial y} d x d y,
\end{gathered}
$$

so that

$$
\int x\left(\frac{\partial u_{X}^{*}}{\partial x} \frac{\partial u_{Y}}{\partial y}-\frac{\partial u_{X}^{*}}{\partial y} \frac{\partial u_{Y}}{\partial x}\right) d x d y=-\int u_{X}^{*} \frac{\partial u_{Y}}{\partial y} d x d y .
$$

Similarly, we have

$$
\int x\left(\frac{\partial u_{Y}^{*}}{\partial y} \frac{\partial u_{X}}{\partial x}-\frac{\partial u_{Y}^{*}}{\partial x} \frac{\partial u_{X}}{\partial y}\right) d x d y=\int u_{Y}^{*} \frac{\partial u_{X}}{\partial y} d x d y,
$$


whence the Cartesian component of vector $\mathbf{r}_{c 2}=\left(\begin{array}{l}x_{c 2} \\ y_{c 2}\end{array}\right)$ may be found:

$$
x_{c 2}=\frac{1}{2 k^{2} I} \int\left(u_{Y}^{*} \frac{\partial u_{X}}{\partial y}-u_{X}^{*} \frac{\partial u_{Y}}{\partial y}\right) d x d y .
$$

Applying Eq. (12) to the $y$ component and performing the same operations give

$$
y_{c 2}=\frac{1}{2 k^{2} I} \int\left(u_{X}^{*} \frac{\partial u_{Y}}{\partial x}-u_{Y}^{*} \frac{\partial u_{X}}{\partial x}\right) d x d y .
$$

Introducing the circular components of the transverse field,

$$
u_{+}=\frac{1}{\sqrt{2}}\left(u_{X}-i u_{Y}\right), \quad u_{-}=\frac{1}{\sqrt{2}}\left(u_{X}+i u_{Y}\right),
$$

one may represent Eqs. (14) and (15) in the form

$$
\begin{aligned}
& x_{c 2}=\frac{i}{2 k^{2} I} \int\left(u_{-}^{*} \frac{\partial u_{-}}{\partial y}-u_{+}^{*} \frac{\partial u_{+}}{\partial y}\right) d x d y, \\
& y_{c 2}=\frac{i}{2 k^{2} I} \int\left(u_{+}^{*} \frac{\partial u_{+}}{\partial x}-u_{-}^{*} \frac{\partial u_{-}}{\partial x}\right) d x d y .
\end{aligned}
$$

Eqs. (16) and (17) describe the spin Hall effect in terms of complex amplitude distributions of circular polarization components of the beam under consideration. They can be represented in a clearer form if one recalls the expression for the first angular irradiance moments of the scalar beam with the complex amplitude $u_{ \pm}[18,19]$ :

$$
\left(\begin{array}{c}
p_{x \pm} \\
p_{y \pm}
\end{array}\right) \equiv \mathbf{p}_{ \pm}=-\frac{i}{k I_{ \pm}} \int u_{ \pm}^{*} \nabla u_{ \pm} d x d y, \quad I_{ \pm}=\int\left|u_{ \pm}\right|^{2} d x d y .
$$

Here the $\mathbf{p}_{ \pm}$vector expresses the mean value of the wavefront inclination with respect to the reference axis $z$, or, what is the same, the tilt of the CG trajectory in the given cross section. Then we have

$$
\begin{aligned}
& x_{c 2}=\frac{1}{2 k}\left(p_{y+} \frac{I_{+}}{I}-p_{y-} \frac{I_{-}}{I}\right), \\
& y_{c 2}=\frac{1}{2 k}\left(p_{x-} \frac{I_{-}}{I}-p_{x+} \frac{I_{+}}{I}\right) .
\end{aligned}
$$

\section{Discussion}

Eqs. (19) and (20) solve the main problem of this study since they describe polarizationdependent part of the transverse position of the beam CG. They have transparent physical consequences, the most important of which are as follows.

(i) The transverse coordinates of the beam CG in any cross section depend upon the spatial distribution of the right- and left-handed polarization components of the incident beam, and the vector $\mathbf{r}_{c 2}$ becomes inverted if the polarization handedness in every point of the beam cross section does. 
(ii) In the beams which are superpositions of the right and left polarised components, the net effect is determined by the difference between the both contributions. Each of the contributions enters with the weight proportional to relative power of the corresponding component. E.g., for the linear polarization the effect disappears when these weights are equal to each other.

(iii) Since the vector $\mathbf{p}_{ \pm}$does not change in the course of free propagation, the polarization-dependent shift of the beam CG should be the same in the all cross sections. Employing just the focal spot for its observation [10] is not a matter of principle, though this may be practical because only in the focal region the beam size is small enough to notice the tiny polarization shift.

(iv) If the beam is polarised homogeneously (i.e., the condition given by Eq. (9) holds true), we have $\mathbf{p}_{+}=\mathbf{p}_{-}=\mathbf{p}$ and Eqs. (19) and (20) reduce to

$$
x_{c 2}=\frac{\sigma}{2 k} p_{y}, y_{c 2}=-\frac{\sigma}{2 k} p_{x},
$$

where $\sigma=\left(I_{+}-I_{-}\right) / I$ is the (constant) polarization helicity. Notice that the CG displacement $\mathbf{r}_{c 2}$ is orthogonal to the $\mathbf{p}$ vector: $\left(\mathbf{p} \cdot \mathbf{r}_{c 2}\right)=0$. The case illustrated by Fig. 1 (the beam profile is symmetric with respect to the $x$ axis but asymmetric with respect to the $y$ axis) corresponds to $p_{y}=0$. Then, in application to the incident beam profile employed in Ref. [9], the first of Eqs. (18) and the second of Eqs. (21) yield the same result as the direct calculations of Ref. [9] based on the explicit expressions for the complex amplitude distribution.

(v) Eqs. (19) and (20) also disclose the nature of the incident beam asymmetry necessary for the effect discussed: it reduces to the requirement that $p_{x \pm}$ and/or $p_{y \pm}$ should differ from zero. If the beam is symmetric 'in itself' (e.g., a circular Gaussian beam), this asymmetry can appear from the beam inclination (see Fig. 2). This situation reminds the spin-orbit and orbit-orbit effects associated with the oblique section of a light beam $[13,20]$ but here, due to supposed paraxiality, the inclination angle $\theta$ is much less than the beam divergence - quite opposite to the conditions accepted in $[13,20]$.

Under conditions prescribed by Fig. 2, the complex beam amplitude contains the phase factor $\exp (\mathrm{i} k \theta y)$ and, according to (18), we have $p_{y}=\theta$. In agreement with Eqs. (21), this entails

$$
x_{c 2}=\frac{\sigma}{2 k} \theta \approx \frac{\sigma}{2 k} \tan \theta .
$$

This result remarkably simulates Eq. (16) from the work [13]; however, Eq. (22) deviates from the formulae of Ref. [13] in the two important aspects. First, the CG definition in [13] differs from our definition (see Eq. (6)) by replacement of the energy density by the longitudinal momentum density. Second, the formulae of Ref. [13] predict the opposite sign of the CG displacement in the geometry of Fig. 2. The latter circumstance is not striking in view of the difference in the CG definitions. However, the visual similarity of 
the analytical representations for the both results is not occasional and reflects the universal geometric nature of the spin Hall effects of light in various systems [1].

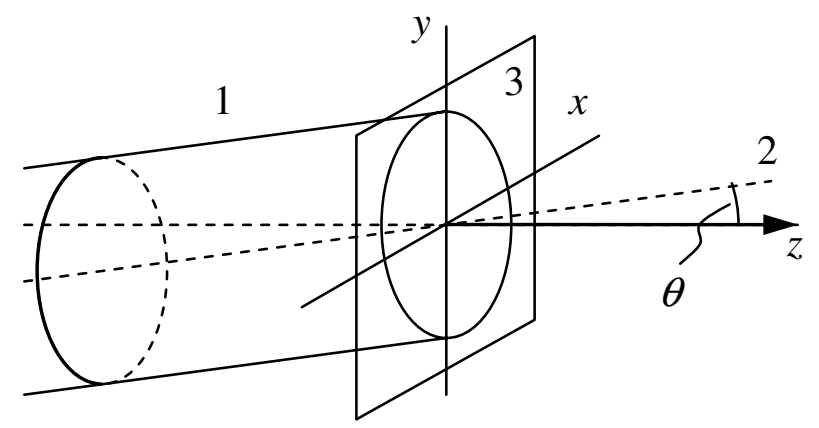

Fig. 2. Geometrical conditions for the case when an inclined incident beam 1 with a tilted propagation axis 2 is analysed in the cross section 3 orthogonal to the $z$ axis. All the other notations are explained in text.

\section{Conclusion}

To finalise our consideration, we emphasise that the shift of the beam CG (6) given by Eqs. (19)-(21) is caused entirely by a polarization-dependent behaviour of the longitudinal field given by Eq. (2). One could suppose that the focusing is essential for the effect because the longitudinal field components increase behind the lens [21]. However, this is not the case, at least as far as the focusing does not change the irradiance moment vector $\mathbf{p}_{ \pm}$(18). In general, the lens with the focal distance $f$ transforms this vector in accordance to the relation $[18,19]$

$$
\mathbf{p}_{ \pm}=\mathbf{p}_{ \pm}^{i}-\frac{\mathbf{r}_{c \pm}^{i}}{f}
$$

where the superscripts " $i$ " denote the quantities associated with \pm -polarised components of the incident collimated beam. In Eq. (23), $\mathbf{r}_{c \pm}^{i}$ means the CG coordinates for the scalar beam model, i.e. it only allows for the transverse field (zero-order of the paraxial approximation) and does not "include" the effect discussed above. Eq. (23) says that if we have $\mathbf{r}_{c \pm}^{i}=0$ (i.e., the "zero-order" CG of the incident beam is situated on the axis $z$ ), the $\mathbf{p}_{ \pm}$vector does not change after focusing. Following from Eqs. (19) and (20), the transverse shift of the focal spot under discussion is then the same as the transverse shift of the incident beam CG. This is an additional confirmation that the use of just the focal spot for observation of the transverse shift $[9,10]$ represents merely a technical expedient. Otherwise, the non-zero $\mathbf{r}_{c \pm}^{i}$ parameter in Eq. (23) is equivalent to the incident beam asymmetry (cf. the half-lens screening in Fig. 1) and the corresponding term $-\mathbf{r}_{c \pm}^{i} / f$ expresses the focused beam inclination analogous to the angle $\theta$ in Fig. 2. 
The accuracy of the present analysis is limited by the paraxial approximation. It seems quite appropriate in the light of the above discussion testifying that the effect, in general, is not directly associated with beam focusing. However, removal of the paraxial limitations will be useful in further development. In particular, it will allow considering how the analysed shift of the "energy" CG (6) given by Eq. (22) interplays with the other Hall effects concerned with the longitudinal momentum distribution [13] or originated from the orbital angular momentum [20] of the beam.

\section{Acknowledgement}

This work was supported in part by the Ministry of Education and Science of Ukraine in frame of the budget project No 457/09 (State Registration Number 0109U000942).

\section{References}

1. Bliokh K Y, Niv A, Kleiner V and Hasman E, 2008. Geometrodynamics of spinning light. Nature Photon. 2: 748-753.

2. Liberman V S and Zel'dovich B Y, 1992. Spin-orbit interaction of a photon in an inhomogeneous medium. Phys. Rev. A 46: 5199-5207.

3. Fedoseyev V G, 1991 Lateral displacement of a light beam under reflection and refraction. 2.The displesement calculation. Opt. Spektrosk. 71: 829-834; [Opt. Spektrosk. 71: 992-997].

4. Onoda M, Murakami S and Nagaosa N, 2004. Hall effect of light. Phys. Rev. Lett. 93: 083901.

5. Bliokh K Y and Bliokh Y P, 2007. Polarization, transverse shifts, and angular momentum conservation laws in partial reflection and refraction of an electromagnetic wave packet. Phys. Rev. E 75: 066609.

6. Bliokh K Y, 2009. Geometrodynamics of polarized light: Berry phase and spin Hall effect in a gradient-index medium. J. Opt. A: Pure Appl. Opt. 11: 094009.

7. Marrucci L, Manzo C and Paparo D, 2006. Optical spin-to-orbital angular momentum conversion in inhomogeneous anisotropic media. Phys. Rev. Lett. 96: 163905.

8. Fadeyeva T A, Rubass A F and Volyar A V, 2009. Transverse shift of a higher-order paraxial vortex beam induced by a homogeneous anisotropic medium. Phys. Rev. A 79: 0538115.

9. Baranova N B, Savchenko A Y and Zel'dovich B Y, 1994. Transverse shift of a focal spot due to switching of the sign of circular polarization. JETP Lett. 59: 232-234.

10.Zel'dovich B Y, Kundikova N D and Rogacheva L F, 1994. Observed transverse shift of a focal spot upon a change in the sign of circular polarization. JETP Lett. 59: 766-769.

11. Volyar A, Fadeyeva T, 2000. Nonparaxial Gaussian beam: 2. The splitting of the singular line and optical Magnus effect. Tech. Phys. Lett. 26: 89-96.

12.Zhao Y, Edgar J S, Jeffries G D M, McGloin D and Chiu D T, 2007. Spin-to-orbital angular momentum conversion in a strongly focused optical beam. Phys. Rev. Lett. 99: 073901.

Ukr. J. Phys. Opt. 2011, V12, №1 
Bekshaev A.

13. Aiello A, Lindlein N, Marquardt C and Leuchs G, 2009. Transverse angular momentum and geometric spin Hall effect of light. Phys. Rev. Lett. 103: 100401.

14. Chun-Fang Li, 2008. Representation theory for vector electromagnetic beams. Phys. Rev. A 78: 063831.

15. Haus H. A. Waves and fields in optoelectronics. Englewood Cliffs, New Jersey: Prentice-Hall, Inc. (1984).

16. Bliokh K Y, Alonso M A, Ostrovskaya E A and Aiello A, 2010. Angular momenta and spin-orbit interaction of non-paraxial light in free space. ArXiv:1006.3876v2 [physics.optics].

17. Bekshaev A Ya and Soskin M S, 2007. Transverse energy flows in vectorial fields of paraxial beams with singularities. Opt. Commun. 271: 332-348.

18. Anan'ev Yu A and Bekshaev A Ya, 1994. Theory of intensity moments for arbitrary light beams Opt. Spectr. 76: 558-568.

19. Mejias P M, Martinez-Herrero R, Piquero G and Movilla J M, 2002. Parametric characterization of the spatial structure of non-uniformly polarized laser beams. Prog. Quantum Electron. 26: 65-130.

20. Bekshaev A Ya, 2009. Oblique section of a paraxial light beam: criteria for azimuthal energy flow and orbital angular momentum. J. Opt. A: Pure Appl. Opt. 11: 094003.

21.Nieminen T A, Stilgoe A B, Heckenberg N R and Rubinsztein-Dunlop H, 2008. Angular momentum of a strongly focused Gaussian beam. J. Opt. A: Pure Appl. Opt. 10: 115005 .

Bekshaev A., 2011. Improved theory for the polarization-dependent transverse shift of a paraxial light beam in free space Ukr.J.Phys.Opt. 12: 10-18.

Анотація. Просторовий розподіл поздовжньої компоненти поля циркулярно поляризованого оптичного променя залежить від знаку ииркулярної поляризачії, щчо спричинює бокове зміщення „гравітаційного центру” при перемиканні поляризачії. Запропонована узагальнена теорія цього ефекту, яка демонструє зв'язок вищезгаданого $з$ кутовим моментом променя. Теорія може бути застосована до будь-якого параксіального променя та вказує на те, щзо бокове зміщення є однаковим для всього поперечного перетину променя 